\title{
ERRATUM
}

\section{Helicobacter Pylori: gastric cancer and beyond}

D. Brent Polk and Richard M. Peek, Jr

Nature Reviews Cancer 10, 403-414 (2010)

On page 404 of this Review, the Lewis histo-blood-group antigen $\mathrm{Le}^{\mathrm{b}}$ is stated to be also known as MUC5AC. This is

incorrect. MUC5AC is a carrier of $\mathrm{Le}^{\mathrm{b}}$ on gastric epithelial cells. On page 405 , Le ${ }^{\mathrm{x}}$ is stated to be the same as FUT4 and Le ${ }^{\mathrm{y}}$

the same as FUT3. This is also incorrect. FUT3 is an $\alpha 1,4 / 3$ fucosyltransferase that can add the fucose residue to the GlcNAc

residue on $\mathrm{Le}^{\mathrm{b}}$ structures. FUT4 is an $\alpha 1,3$ fucosyltransferase that adds fucose to GlcNAc residues in type 2 Lewis antigens,

including $L^{x}{ }^{x}, e^{\gamma}$ and sialyl-Le ${ }^{x}$. We would like to thank A. Magalhães and C. A. Reis for alerting us to these mistakes. 